\title{
Comparative effects of acute-methionine loading on the plasma sulfur-amino acids in NAC-supplemented HIV+ patients and healthy controls
}

\author{
Roberto Carlos Burini ${ }^{1} \cdot$ Maria Doroteia Borges-Santos ${ }^{1} \cdot$ Fernando Moreto ${ }^{1} \cdot$ Yong- Ming Yu ${ }^{2}$
}

Received: 25 October 2017 / Accepted: 12 January 2018 / Published online: 1 February 2018

(c) Springer-Verlag GmbH Austria, part of Springer Nature 2018

\begin{abstract}
In this study, an acute overloading of methionine (MetLo) was used to investigate the trassulfuration pathway response comparing healthy controls and HIV+ patients under their usual diet and dietary N-acetyl-L-cysteine (NAC) supplementation. MetLo (0.1 g Met/kg mass weight) was given after overnight fasting to 20 non-HIV+ control subjects (Co) and $12 \mathrm{HIV}+$ HAART-treated patients. Blood samples were taken before and after the MetLo in two different 7-day dietary situations, with NAC (1 g/day) or with their usual diet (UD). The amino acids (Met, Hcy, Cys, Tau, Ser, Glu and Gln) and GSH were determined by HPLC and their inflow rate into circulation (plasma) was estimated by the area under the curve (AUC). Under UD, the HIV+ had lower plasma GSH and amino acids (excepting Hcy) and higher oxidative stress (GSSG/GSH ratio), similar remethylation (RM: Me/Hcy + Ser ratio), transmethylation (TM; Hcy/Met ratio) and glutaminogenesis (Glu/Gln ratio), lower transsulfuration (TS: Cys/Hcy + Ser ratio) and Cys/Met ratio and, higher synthetic rates of glutathione (GG: GSH/Cys ratio) and Tau (TG: Tau/Cys ratio). NAC supplementation changed the HIV pattern by increasing RM above control, normalizing plasma Met and TS and, increasing plasma GSH and GG above controls. However, plasma Cys was kept always below controls probably, associatively to its higher consumption in GG (more GSSG than GSH) and TG. The failure of restoring normal Cys by MetLo, in addition to NAC, in HIV + patients seems to be related to increased flux of Cys into GSH and Tau pathways, probably strengthening the cell-antioxidant capacity against the HIV progression (registered at http://www.clinicaltrials.gov, NCT00910442).
\end{abstract}

Keywords HIV+ patients $\cdot$ Methionine-load test $\cdot$ Thiol redox system $\cdot$ Cysteine metabolism

\section{Introduction}

Previously, we described that plasma reduced glutathione (GSH) was decreased in HIV+ patients compared to healthy controls subjects and, GSH deficiency would be either due to a deficient synthesis and/or a lower GSSG (glutathione oxidized form)-GSH conversion (Borges-Santos et al. 2012). GSH is synthesized de novo within all cells (Kozich

Roberto Carlos Burini

burini@fmb.unesp.br

1 Department of Public Health, Center for Nutritional and Physical Exercise Metabolism, School of Medicine, UNESP-Sao Paulo State University, Campus Botucatu, Botucatu, Sao Paulo, Brazil

2 Department of Surgery, Harvard Medical School, Massachusetts General Hospital, Shriners Burns Hospital, Boston, MA, USA et al. 1995), but plasma GSH arises largely from the liver. The lower plasma GSH follows the lack of its components, L-cysteine (Cys), L-glutamic acid (Glu), and L-glycine (Gly), which in turn parallel the lower levels of their precursors L-methionine (Met) and L-serine (Ser) (for Cys) and L-glutamine (Gln) (for Glu) (Fermo et al. 1995; Bogden et al. 2000; Patrick 2000; Lanzillotti and Tang 2005; Jones et al. 2006; Burini et al. 2015). In a previous publication, it was shown that HIV+ patients had their plasma GSH increased to the control levels by receiving either Cys (by the oral intake of N-acetyl-L-cysteine, NAC) or Gln. However, even with NAC supplementation, plasma Cys level was kept lower than controls (Borges-Santos et al. 2012).

Intracellular Cys availability is believed to be the rate-limiting precursor for GSH synthesis (Lyons et al. 2000; Badaloo et al. 2002; Jackson et al. 2004). Whole-body Cys is maintained in balance between formation and degradation with the main byproducts of Cys being taurine (Tau) and sulfate 
(Griffith 1987). Tau has systemic anti-inflammatory actions and, inside phagocytes, it acts as protective antioxidant (Burini et al. 2018). Additionally, it is described that Tau enables antigen-presenting cells to promote the proliferation of $\mathrm{T}$ lymphocytes (Dröge et al. 1991).

It has been considered that a higher urinary-sulfate output by HIV+ patients could be due to the fact that muscle excretes Cys to spare protein nitrogen (forming Gln in the liver) and restrain the body-protein catabolism (Dröge and Holm 1997). By the muscle excreting Cys to the liver (Sbrana et al. 2004) the organism restrains the whole body-protein catabolism because Cys catabolism generates an increased formation of sulfate and protons which down-regulates the rate of urea production (Viñ et al. 2001) allowing Gln synthesis and, therefore, nitrogen conservation, while the generated sulfate is excreted by urine. Even asymptomatic HIV-positive persons reveal, on average, a massive daily loss of sulfur, and may explain the widely observed decrease in cyst(e)ine and GSH levels. This rate of sulfur loss is not ameliorated by highly active antiretroviral therapy and may contribute to treatment failure (Dröge and Breitkreutz 1999).

In pathological conditions such as $\mathrm{HIV}+$, Cys is considered conditionally indispensable (Dröge et al. 1991; Borges-Santos et al. 2012). The endogenous sources of Cys are half derived from Met, through the transsulfuration (TS) pathway and half derived from GSH breakdown (Burini et al. 2013). Only the sulfur atom from Met is transferred by TS to Cys; the carbon skeleton of Cys is donated by Ser (Stipanuk 2004). Hence, in the fasting state, Cys is maintained mostly by the GSH turnover (Bogden et al. 2000), while after Met loading (MetLo), Cys might originate, mostly from the TS (Stipanuk 2004) by de novo synthesis.

Generally, the most extensive approach to increase the cell pool of GSH is by improving Cys availability through oral agents such as NAC, lipoic acid, cysteamine, and 2-oxothiazolidine 4-carboxylate (Grimble 2005). NAC has long been considered as a potent antioxidant, primarily as a pro-drug for GSH (Zhou et al. 2015). From previous analysis, we had found that, despite normalizing GSH, the NAC supplement was unable to restore the normal levels of plasma Cys, which raises the hypothesis of TS failure in HIV+.

Thus, the present paper aimed to explore the Cys metabolism, particularly the TS pathway of Met by analyzing the plasma levels of Cys and its byproducts Tau and GSH in response to the MetLo without and with the presence of NAC supplementation, comparatively in healthy controls and HIV+ patients.

\section{Materials and methods}

\section{Subjects}

This is a complementary study of other already detailed elsewhere (Borges-Santos et al. 2012). From the 28 HIV+ patients attended at the Infectious Disease ward of the Clinical Hospital, 12 patients fulfilled the inclusion criteria of having all the data before and after the interventions. The group of 12 HIV+ patients ( 6 men and 6 women), 22-45 years, was clinically and laboratory diagnosed with viral load (by ELISA and Western Blot) and $\mathrm{CD}_{4}{ }^{+}$and $\mathrm{CD}_{8}{ }^{+}$lymphocyte counts (by flow cytometry). HIV+ patients were under highly active antiretroviral treatment for at least 1 year with HIV protease inhibitors (indinavir: $800 \mathrm{mg}$ twice daily, $n=10$, or ritonavir $600 \mathrm{mg}$ twice daily, $n=2$ ) combined with two nucleoside analogs (zidovudine: $250 \mathrm{mg}$ plus lamivudine: $150 \mathrm{mg}$ twice daily, $n=11$ or lamivudine: $150 \mathrm{mg}$ plus stavudine: $40 \mathrm{mg}$ twice daily, $n=1$ ). The selected patients were without renal or liver failure and not taking any form of B vitamins or sulfur-amino acids. The patients group was compared to a clinically healthy control group of 20 adults (10 men and 10 women), 20-59 years, negative for HIV, recruited from the UNESP Clinical Hospital to participate in this study. The study protocol was approved by ethical committees from Sao Paulo State University and Sao Paulo University and all procedures were performed according to the Declaration of Helsinki. All subjects had a signed consent form.

\section{Experimental design}

After baseline clinical assessments, the two groups had the fasting blood drawn before and after seven consecutive periods receiving either their usual control diet alone (UD) or added a dietary supplement of NAC at $1 \mathrm{~g} /$ day. Immediately after the overnight-fast blood drawn, they all submitted an oral MetLo ( $0.1 \mathrm{~g}$ Met $/ \mathrm{kg}$ body wt) with blood samples taken 2 and $4 \mathrm{~h}$ after the MetLo.

\section{Reagents and equipment}

The reagents ethylene diamine tetraacetate (EDTA), $n$-ethylmaleimide (NEM), $o$-phtaldialdehyde (OPA), tri- $n$-butylphosphine (TBP), dithiothreitol (DTT), ammonium7-fluorobenzo-2-oxa-1,3-diazole-4-sulphonate (SBD-F) and analytical standards were purchased from SigmaAldrich $^{\circledR}$. Sulphosalicylic acid (SSA), perchloric acid and methanol and acetonitrile HPLC-analytical grade were purchased from Merck-Millipore ${ }^{\circledR}$. 
The high-performance liquid chromatography (HPLC) system consisted of a LC10AD Shimadzu apparatus, equipped with two pumps, degasser DGU-10B, sample auto-injector SIL10ADVP, column-oven CTO-10AC and UV-detector SPD-20AV. Mobile phases were pre-filtered using membrane filters $0.45 \mu \mathrm{m}$ (HNWPO4700, MerckMillipore $\left.{ }^{\circledR}\right)$. Samples were also filtered with LCR-PTFE syringe filters $\left(0.22 \mu \mathrm{m}\right.$, Merck-Millipore $\left.{ }^{\circledR}\right)$ before HPLC injection. Analytical columns for all analysis were purchased from Waters Corporation (Spherisorb ${ }^{\circledR}$, ODS-2, $150 \times 4.5 \mathrm{~mm})$.

\section{Laboratory analysis}

Blood samples were collected into two EDTA containing tubes. In one tube, PBS was added and into another, NEM was added (10 mM in PBS). Both tubes were centrifuged for plasma obtaining. Plasma from PBS-containing tube was used for amino acids and total glutathione measurements. Plasma from NEM-containing tube was used for GSSG measurements.

For amino acids measurement, plasma was first treated with SSA 30\%, centrifuged and supernatant was derivatized with OPA before injecting it into the HPLC system (Qureshi and Qureshi 1989).

Total plasma Cys and L-homocysteine (Hcy) were measured in a separated analysis. Plasma was treated with TBP and then derivatized with SBD-F before injecting it into the HPLC system (Ubbink et al. 1991).

Total glutathione and GSSG levels were measured in plasma from PBS-containing tube and plasma from NEMcontaining tube, respectively. Samples were submitted to reduction with DTT, precipitation with perchloric acid and derivatization with OPA before injecting it into the HPLC system. GSH (reduced form) levels were estimated by subtracting GSSG values from total glutathione values (Paroni et al. 1995).

The plasma Hcy/Met ratio was defined arbitrarily as "transmethylation" (TM), Met/Hcy as "remethylation" (RM), Cys/Hcy + Ser as "transsulfuration" (TS), Tau/Cys as "taurine-genesis" (TG), GSH/Cys as "glutathione-genesis" (GG) and GSSG/GSH was used as oxidative stress indicator.

\section{Data processing and statistical analysis}

The area under the curve (AUC) was calculated for sulfuramino acids and glutathione. The Kolmogorov/Smirnov test was applied to check sample variability in all variables. Parametric variables are expressed as mean \pm S.D, whereas non-parametric variables are expressed as median (p25-p75 interquartile). The difference between groups and diets was compared by the $t$ test (for parametric variables) or Wilcoxon's test (for non-parametric variables). The analyses were performed using the SAS package v. 8.01 (SAS Institute, Inc., Cary, NC, USA) and statistical significance was set at $p<0.05$.

\section{Results}

Under UD, all amino acids and GSH were lower in HIV+ patients than controls after MetLo and also at baseline (excepting Hcy) (Table 1). However, HIV+ patients were similar to controls in all indices without Cys participation (TM, RM and Glu/Gln), lower than controls for Cys/Met and TS and higher than controls for GSSG/GSH, TG and GG. Similarly, after MetLo (AUC), the HIV+ group followed the same baseline pattern in both dietary regimen, UD and NAC (Table 1 and Fig. 1).

The NAC supplementation matched the patient's plasma Met and Hcy to control's values, keeping the other amino acids and GSH lower than the control group. On the other hand, NAC supplementation did not alter significantly the UD pattern of TM, TG, Cys/Met and Gln/Cys (Table 1 and Fig. 1). However, with NAC, TS paired to the control values. Besides this, the booster effect of NAC was seen also by increasing the RM over the control values. Both NAC effects, on RM and TS, were neutralized by the MetLo (Table 1 and Fig. 1). Hence, NAC supplementation promoted a deep increase of RM, TS and GG at baseline, but only GG occurred due to the presence of MetLo (Table 1 and Fig. 1).

Thus, under UD, HIV+ group presented TM, RM and Glu/Gln values similar to controls irrespective of the presence MetLo (Table 1). Under NAC, these similarities were extended also for TS, and the differences between groups, increased for RM and GG and, decreased for Glu/Gln (Table 1 and Fig. 1). The MetLo attenuated (GG) or even neutralized (RM, TS, Gln/Cys and Glu/Gln) the NAC effects (Table 1 and Fig. 1). In general, HIV+ always presented higher than controls TG and GG irrespective of the presence of NAC and MetLo; MetLo magnified even more higher GG effect of NAC. Overall, the lower than control TS found in HIV+ was corrected by the NAC supplementation (Fig. 1). Therefore, it seems that Cys stayed lower in HIV irrespective of supplied sources (NAC and Met), which would be caused by higher consumption (higher GSH, Tau and Gln generation) rather than lower synthesis (TS) of Cys.

\section{Discussion}

The acute MetLo is an in vivo test described as useful to assay the functionality of the Met cycle (Di Giuseppe et al. 2010) particularly the TS pathway where Cys might originate from the de novo synthesis (Stipanuk 2004). Both 
Table 1 Plasma amino acids, total glutathione $(\mu \mathrm{mol} / \mathrm{L})$, and its rations in healthy controls (Control) and HIV+ patients (HIV), before (B) and after methionine load (AUC), under usual diet (UD) and oral $N$-acetylcysteine supplementation (NAC)

\begin{tabular}{|c|c|c|c|c|c|}
\hline & & \multicolumn{2}{|l|}{ UD } & \multicolumn{2}{|l|}{ NAC } \\
\hline & & Control & HIV & Control & HIV \\
\hline \multirow[t]{2}{*}{ Met } & B & $26.8(24.1-38.7)$ & $16.7(15.1-26.6)^{*}$ & $26.3(22.8-39.6)$ & $21.3(19.3-31.8)$ \\
\hline & AUC & $1811 \pm 516$ & $1459 \pm 430 * *$ & $1711 \pm 483$ & $1441 \pm 390$ \\
\hline \multirow[t]{2}{*}{ Hcy } & $\mathrm{B}$ & $11.2(9.9-19.5)$ & $9.7(8.5-11.2)$ & $14.4(11.7-15.5)$ & $12.9(10.4-13.6)$ \\
\hline & AUC & $118 \pm 51.4$ & $60.9 \pm 6.2^{* *}$ & $132 \pm 26.6$ & $81.5 \pm 18.0^{* *}$ \\
\hline \multirow[t]{2}{*}{ Ser } & B & $106(101-115)$ & $86.9 \pm 20.1^{*}$ & $110(100-120)$ & $60.3(54.7-65.1)^{*}$ \\
\hline & AUC & $411 \pm 30.8$ & $275 \pm 54.9 * *$ & $413 \pm 35.5$ & $228 \pm 50.2 * *$ \\
\hline \multirow[t]{2}{*}{ Cys } & B & $317(268-593)$ & $157(151-166)^{*}$ & $534 \pm 53.9$ & $251 \pm 31.6^{*}$ \\
\hline & AUC & $1537 \pm 643$ & $620 \pm 30.8^{* *}$ & $1887 \pm 272$ & $1080 \pm 113^{* *}$ \\
\hline \multirow[t]{2}{*}{ Tau } & B & $62.3(61.3-66.1)$ & $48.4(45.9-51.0)^{*}$ & $71.3(61.1-77.3)$ & $57.2(54.8-61.1)^{*}$ \\
\hline & AUC & $443 \pm 28.4$ & $379 \pm 51.6^{* *}$ & $486 \pm 51.5$ & $523 \pm 40.5^{*}$ \\
\hline \multirow[t]{2}{*}{ GSH } & B & $8.6(7.9-10.0)$ & $4.9 \pm 0.7^{*}$ & $8.5 \pm 0.9$ & $9.2 \pm 0.9^{*}$ \\
\hline & AUC & $35.8 \pm 6.0$ & $20.4 \pm 1.5^{* *}$ & $34.5 \pm 4.8$ & $40.1 \pm 2.1 * *$ \\
\hline \multirow[t]{2}{*}{ Hcy/Met (TM) } & B & $0.41(0.33-0.57)^{\mathrm{a}}$ & $0.52(0.37-0.67)^{\mathrm{a}}$ & $0.46(0.36-0.57)^{\mathrm{a}}$ & $0.50(0.38-0.68)^{\mathrm{a}}$ \\
\hline & AUC & $0.57(0.46-0.78)^{\mathrm{a}}$ & $0.65(0.45-0.79)^{\mathrm{a}}$ & $0.66(0.57-0.81)^{\mathrm{a}}$ & $0.67(0.55-0.82)^{\mathrm{a}}$ \\
\hline \multirow[t]{2}{*}{ Met/Hcy (RM) } & B & $2.56 \pm 1.22^{\mathrm{a}}$ & $2.06 \pm 0.68^{\mathrm{a}}$ & $2.29 \pm 0.86^{\mathrm{a}}$ & $2.11 \pm 0.87^{\mathrm{a}}$ \\
\hline & AUC & $17.6(12.8-21.8)^{\mathrm{a}}$ & $15.1(12.6-22.4)^{\mathrm{a}}$ & $15.0(12.4-17.5)^{\mathrm{a}}$ & $14.9(12.2-18.4)^{\mathrm{a}}$ \\
\hline \multirow[t]{2}{*}{ Cys/Hcy + Ser (TS) } & B & $3.35 \pm 1.28^{\mathrm{b}}$ & $1.73 \pm 0.57^{\mathrm{a}}$ & $4.33 \pm 0.54^{\mathrm{c}}$ & $3.50 \pm 0.64^{\mathrm{bc}}$ \\
\hline & AUC & $2.05(1.82-2.37)^{\mathrm{b}}$ & $0.92(0.78-1.19)^{\mathrm{a}}$ & $2.89(1.85-3.11)^{\mathrm{b}}$ & $2.67(1.23-3.07)^{\mathrm{b}}$ \\
\hline \multirow[t]{2}{*}{ Tau/Cys (TG) } & B & $0.18 \pm 0.07^{\mathrm{b}}$ & $0.31 \pm 0.03^{\mathrm{d}}$ & $0.13 \pm 0.02^{\mathrm{a}}$ & $0.23 \pm 0.04^{c}$ \\
\hline & AUC & $0.28 \pm 0.04^{\mathrm{a}}$ & $0.61 \pm 1.68^{\mathrm{ab}}$ & $0.26 \pm 0.19^{\mathrm{ab}}$ & $0.48 \pm 0.36^{\mathrm{b}}$ \\
\hline \multirow[t]{2}{*}{$\mathrm{GSH} / \mathrm{Cys} \times 100(\mathrm{GG})$} & $\mathrm{B}$ & $2.7(1.7-3.0)^{\mathrm{a}}$ & $3.1(2.8-3.4)^{\mathrm{b}}$ & $1.6(1.5-1.7)^{\mathrm{a}}$ & $3.7(3.3-4.0)^{\mathrm{c}}$ \\
\hline & AUC & $2.3 \pm 0.9^{\mathrm{a}}$ & $3.3 \pm 1.4^{\mathrm{b}}$ & $1.8 \pm 0.8^{\mathrm{a}}$ & $3.7 \pm 1.9^{\mathrm{b}}$ \\
\hline \multirow[t]{2}{*}{ GSSG/GSH } & B & $7.59(4.8-9.8)^{\mathrm{a}}$ & $29.6 \pm 6.1^{\mathrm{b}}$ & $6.92(5.96-7.7)^{\mathrm{a}}$ & $18.5 \pm 3.0^{\mathrm{c}}$ \\
\hline & AUC & $0.34 \pm 0.23^{\mathrm{a}}$ & $0.87 \pm 0.20^{\mathrm{b}}$ & $0.27 \pm 0.16^{\mathrm{a}}$ & $0.55 \pm 0.19^{\mathrm{b}}$ \\
\hline
\end{tabular}

$B$ baseline, $A U C$ area under the curve after methionine load ( $0.1 \mathrm{~g} \mathrm{Met} / \mathrm{kg}$ body wt), $T M$ transmethylation, RM remethylation, $T S$ transsulfuration, $T G$ taurine-genesis, $G G$ glutathione-genesis, $G S S G / G S H$ as oxidative stress indicator

*Different from control group, $p<0.05$; ** different from control group, $p<0.001$; small letters mean statistical difference $(p<0.05): \mathrm{a} \neq \mathrm{b} \neq \mathrm{c} \neq \mathrm{d}$ chronic and acute MetLo tests are useful to assay the TS reactions (Giuseppe et al. 2010). The rationale of the test is that Cys is not a precursor of Met, because of the irreversibility of the cystathionine synthase reaction (Rose 1938).

Met is considered an indispensable amino acid, because mammals cannot synthesize its $\mathrm{C}$ skeleton. The first step in the metabolism of Met is its conversion to the intermediate, S-adenosylmethionine (AdoMet), a donor of methyl groups for numerous methylation reactions (Stipanuk 2004). The other product of TM of Met is S-adenosylhomocysteine (AdoHcy), which is hydrolyzed to adenosine and Hcy. The present data showed similar plasma Hcy and TM between patients and controls, irrespective of the MetLo.

Hcy is at a branch point and can be either remethylated to Met by the methionine synthase or it can be condensed with Ser to form L-cystathionine through the TS pathway due to the effect of cystathionine beta-synthase (CBS). CBS is activated by AdoMet formed in the TM pathway of Met to Hcy. The TS sequence is completed by a reaction catalyzed by gamma-cystathionase, which is responsible for the cleavage of L-cystathionine to yield Cys, alpha-ketobutyrate and $\mathrm{NH}_{4}{ }^{+}$. Hence, TS is an essential pathway allowing the synthesis of Cys from Met therefore, Met: Cys ratio can be taken as marker to evaluate the S-amino acid metabolism involved in Cys generation from Met (Viñ et al. 2001).

Decreased Cys/Met ratio means reduced conversion of Met to Cys. The main removal of synthesized Hcy is by Met synthase (Burini et al. 2013). As the TM, the RM also remained similar between the two groups. Keeping Hcy seems tactical for the HIV+ due to its pro-oxidant properties (Burini et al. 2013) and Hcy level is associated with status of oxidative stress (Tousoulis et al. 2008). In the present case, the resulted pro-oxidant status, can be seen in GSH-GSSG redox ratio, favoring GSSG, might be related to a more oxidant redox status promoted by changed Cys/L-cystine couples (Jones 2004).

Along with RM, another pathway to remove Hcy is by TS. In this pathway, Hcy is removed by combination with 

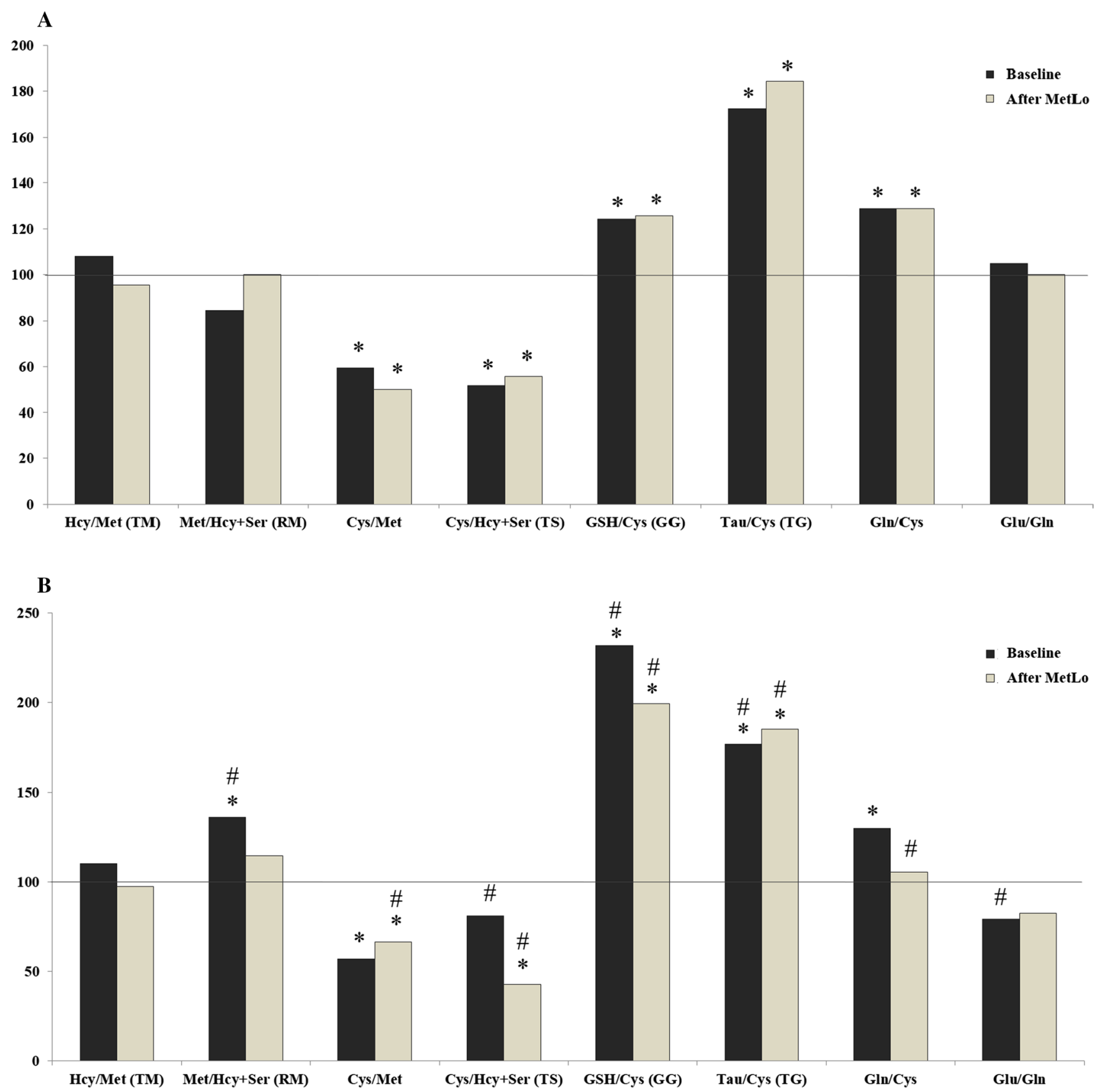

Fig. 1 HIV+ Differences (\%) related to control values at baseline and after MetLo in both dietary conditions, usual diet (a) and NACsupplemented (b). TM transmethylation, $R M$ remethylation, $T S$ trans- sulfuration, $T G$ taurine-genesis, $G G$ glutathione-genesis. ${ }^{*} p<0.05$ $(\mathrm{HIV} \neq \mathrm{Co}) ; \# p<0.05$ (NAC $\neq \mathrm{UD})$
Ser (van der Griend et al. 2002) to form Cys after cleavage of cystathionine (Garlick 2006). Our HIV+ patients presented a reduced Cys/Met ratio (40.4\%) as well as TS (48.4\%) meaning reduced formation of Cys from Met probably by lower TS pathway. This lower conversion of Met to Cys, at baseline, was confirmed by MetLo. As already mentioned, this low TS rate would favor HIV+ once in the presence of normal RM, it keeps Hcy and, consequently the pro-oxidant milieu, convenient for the virus survival (Burini et al. 2013).

TS is controlled by CBS that is activated by AdoMet formed in the TM (in this case, not affected by HIV+) and gamma-cystathionase. In an experimental in vivo inhibition of the TS pathway by inactivating gamma-cystathionase, blood concentration of L-cystathionine was greater than controls and L-cystine was unchanged consequently, 
the Met/L-cyst(e)ine ratio was significantly higher and, liver GSH concentration was significantly lower than values of control livers (Triguero et al. 1997). Patients reported here showed increased Met/L-cyst(e)ine ratio, similar to the one found in this gamma-cystathionase experimental inhibition. But, differently, in the present patients, the reduced TS and plasma GSH were followed by low Cys. Hence, these set of data probably meant that lower TS pathway was not the only cause of lowering plasma Cys. It might be complemented by a lower contribution of Cys from GSH recycling, as suggested here, by the found higher GSH/Cys ratio.

Besides TS from Met, already reduced in our HIV+ patients, another source of Cys would be the GSH breakdown, responsible for half of plasma Cys (Burini et al. 2013). The possibility of a lower origin of Cys from GSH breakdown exists, because quantitatively, plasma level of GSH was always lower than controls, irrespective of MetLo. Moreover, HIV+ patients also presented higher GSH/Cys (GG) and GSSG/Cys ratios, which would favor GSH maintenance rather than GSH breakdown for Cys supplying. Thus, lower plasma Cys could result in lower endogenous contribution of both sources, Met (TS pathway) and GSH breakdown.

Regarding a possible higher HIV+-induced Cys catabolism, it is known that, in post-absorptive state, muscle releases Cys that is taken up by the liver. Therefore, the basal Cys level is regulated primarily by the balance of normal post-absorptive skeletal muscle protein catabolism and liver uptake (Viñ et al. 2001). The decreased blood Gln/L-cyst(e) ine ratio has been proposed as a good marker of an impaired hepatic L-cyst(e)ine catabolism (Dröge and Holm 1997). The present HIV+ data showing 29\% higher Gln/Cys (at baseline and under MetLo) along with similar Glu/Gln to the controls would suggest a HIV+ condition of normal glutaminogenesis (Glu/Gln) in a fair controlled whole-body catabolism (higher Gln/Cys). Moreover, considering that Met/Lcyst(e)ine and Gln/L-cyst(e)ine ratios can be taken as good markers to evaluate the S-amino acid metabolism (Viñ et al. 2001), we can assume to have had more metabolic problems with Cys than with Met and Gln.

Thus, the decreased plasma Cys was not associated to its higher breakdown and, rather might be due to a possible higher utilization in GSH synthesis. Actually, when looking at the metabolic fate of Cys, we see higher synthetic activity of both antioxidant byproducts, GSH (GG) and Tau (TG) as well as in an N-conservation index (Gln/Cys). As in TS pathway, the GG, TG and Gln/Cys patterns seem physiologically stable and not influenced by the acute Met loading.

In this study, plasma GSH was lower than controls associated with lower plasma Cys and higher GG denoting higher consumption of Cys for formation of either GSH (GSH/ Cys), Tau (Tau/Cys) and Gln (Gln/Cys).
A second possible cause for the lower plasma level of GSH in our patients would be the enhanced entrapment of GSH in its oxidized form (GSSG) (Nguyen et al. 2014) as seen by the increased GSSG/GSH ratio at 3.9× (at baseline) and 2.56× (under AUC). The GSSG is converted back to reduced GSH by the enzyme GSH reductase, in a riboflavindependent reaction (Meister 1988). Thus, it seems that our HIV-infected patients presented plasma pattern suggestive of deficient Cys synthesis and decreased GSH regeneration (from GSSG), which contributed to their GSH deficiency (Nguyen et al. 2014). Both defects (GSH synthesis and regeneration) can be overcome with shot-time supplementation with precursors (Sekhar et al. 2011; Borges-Santos et al. 2012).

In the experimental in vivo inhibition of the TS pathway (Triguero et al. 1997), the administration of NAC reversed the changes in liver GSH. The proposed mechanism of direct conversion would be through deacetylation of NAC to Cys by acylase I, widely expressed in the cytosol. Alternatively, there is an indirect mechanism in which NAC modulates plasma and tissue protein-bound Cys levels through thiol exchange. In this pathway, NAC increases Cys concentration through thiol exchange with oxidized/bound forms of Cys (e.g., protein-bound) (Zhou et al. 2015). Both mechanisms might workout in our case. In fact, when given to our patients, NAC led to an increased level of GSH by increasing RM, TS and GG. In parallel, NAC normalized the plasma levels of Met and Hcy.

The effect of NAC supplementation on plasma Met was expected once Cys has a methionine-sparing action which is believed to occur by reducing methionine breakdown through TS (Fukagawa 2006). By leading to higher RM and TS and keeping TM unchanged, the NAC-supplemented HIV+ allowed the Hcy levels to stay similar to control levels and this condition favored both the invader (by offering Hcy) and the host (by increasing RM and sparing Met). By adding simultaneously Cys (NAC) and Met (MetLo), also the RM value was normalized to controls, maybe by improving Met (for the host) and Hcy (for the virus). Thus, from the present data, by keeping GG always higher than controls, irrespective of Met (MetLo), it seems that Cys (NAC) is really the enhancer of GSH formation in HIV+.

Although not changing the pattern of the control group, NAC supplementation increased the patient's TS and consequently, both groups became similar. Hence, it seems that TS is kept decreased in HIV+ to keep adequate Hcy levels and, exogenous sources of Cys (and spared Met) are necessary to restore normal TS. However, providing Cys to HIV patients, GG increases and plasma levels of GSH surpass the controls, denoting a clear purpose for Cys utilization. This fact controversies the theory that the conversion rate of NAC to Cys by deacetylation is not adequate to maintain single Cys levels for GSH biosynthesis and, findings from stable isotopes indicated 
that endogenous Cys rather than exogenous Cys would be the major source for GSH synthesis, after NAC administration (Raftos et al. 2007). Though, alternatively, it is proposed that NAC modulates indirectly plasma and tissue protein-bound Cys levels, through thiol exchange. Consequently, NAC increases Cys concentration through thiol exchange with oxidized/bound forms of Cys (e.g., protein bound) (Zhou et al. 2015). In vitro plasma study has shown that unbound Cys in plasma was quickly released from Cys bound peptides (e.g., GSSG) when incubated with increasing NAC concentrations (Radtke et al. 2012).

Although the Cys-Cystine thiol exchange form have not been assayed here, the present data showed that HIV+ GSSG/ GSH ratio decreased from $3.9 \times$ at baseline to $2.7 \times$ under NAC supplementation, clearly showing a deficient GSH restoration from GSSG. The decreasing of oxidative stress (GSSG/GSH ratio) can be attributed to NAC actions (Nguyen et al. 2014).

Besides increasing GSH (and decreasing GSSG) and normalizing plasma Met, Hcy and the TS, the normal plasma Cys was never restored to the healthy control levels in HIV+ patients with NAC and MetLo. Thus, the two key findings can be ascertained from our NAC-supplemented HIV+, first, the enhancement of exogenous Cys in GSH synthesis (overcoming basal TS limitation) and second, the NAC limitation in fully regenerating GSH from GSSG.

Different from GSH, the Tau synthesis (TG) was higher than controls irrespective of either NAC or MetLo. Tau acts as protective antioxidant and anti-inflammatory agent (Angelini et al. 2002; Burini et al. 2018).

Thus, both products of Cys (GSH and Tau) seem important for the host defense against HIV-induced oxidative stress, and both are replenished under NAC supplementation, although both replenishments were accomplished associatively with low plasma levels of Cys. Hence, it was confirmed that Cys may be considered conditionally as an indispensable amino acid in pathological conditions such as HIV+ (Dröge et al. 1991; Borges-Santos et al. 2012), because it is the precursor of GSH-, Tau-, and Gln-genesis controller (Griffith 1987). Presently, the low plasma Cys found in HIV seems to be a consequence of reducing TS pathway and higher GSH and Tau formation. The NAC supplementation of HIV+ patient led to an improvement of TS, increased GG and plasma GSH (also Met and Hcy) as well as TG probably towards an improvement of the cellantioxidant status. Nevertheless, no additional contribution of MetLo was detected beyond the NAC supplementation.

\section{Conclusion}

Exogenous Cys (NAC) even in the presence of Met (MetLo) failed to restore control levels of Cys besides increasing its formation pathways and having normalized the levels of either its precursors (Met, Hcy and GSH) and byproducts (GSH and Tau). HIV+ patients seem to use the sulfur pathways primarily fluxing Cys into GSH and Tau pathways to strengthen the cell-antioxidant capacity against the HIV progression.

Acknowledgements To the Brazilian institutions FAPESP and CNPq for the research funding and fellowships.

\section{Compliance with ethical standards}

Conflict of interest The authors declare no conflicts of interest.

Ethical approval All procedures performed in studies involving human participants were in accordance with the ethical standards of the institutional and/or national research committee and with the 1964 Helsinki declaration and its later amendments or comparable ethical standards.

Informed consent Informed consent was obtained from all individual participants included in the study.

\section{References}

Angelini G, Gardella S, Ardy M et al (2002) Antigen-presenting dendritic cells provide the reducing extracellular microenvironment required for T lymphocyte activation. Proc Natl Acad Sci USA 99:1491-1496. https://doi.org/10.1073/pnas.022630299

Badaloo A, Reid M, Forrester T et al (2002) Cysteine supplementation improves the erythrocyte glutathione synthesis rate in children with severe edematous malnutrition. Am J Clin Nutr 76:646-652. https://doi.org/10.3945/ajcn.111.024323.INTRODUCTION

Bogden JD, Kemp FW, Han S et al (2000) Status of selected nutrients and progression of human immunodeficiency virus type 1 infection. Am J Clin Nutr 72:809-815

Borges-Santos MD, Moreto F, Pereira PCM et al (2012) Plasma glutathione of HIV+ patients responded positively and differently to dietary supplementation with cysteine or glutamine. Nutrition 28:753-756. https://doi.org/10.1016/j.nut.2011.10.014

Burini RC, Moreto F, Borges-Santos MD, Yu YM (2013) Plasma homocysteine and thiol redox states in HIV+ patients. In: McCully K (ed) Homocysteine: biosynthesis and health implications, 1st edn. Nova Science, New York City, pp 151-164

Burini RC, Borges-Santos MD, Moreto F, Yu YM (2015) Plasma antioxidants and glutamine supplementation in HIV. In: Rajendram R, Preedy V, Patel V (eds) Glutamine in clinical nutrition, Nutrition and health. Humana Press, New York

Burini RC, Borges-Santos MD, Moreto F, Ming-Yu Y (2018) Taurine and oxidative stress in HIV. In: Preedy V, Watson RR (eds) HIV/ AIDS: Oxidative stress and dietary antioxidants. Elsevier, Amsterdam, pp 170-179

Di Giuseppe D, Ulivelli M, Bartalini S et al (2010) Regulation of redox forms of plasma thiols by albumin in multiple sclerosis after fasting and methionine loading test. Amino Acids 38:1461-1471. https://doi.org/10.1007/s00726-009-0350-8

Dröge W, Breitkreutz R (1999) N-acetyl-cysteine in the therapy of HIV-positive patients. Curr Opin Clin Nutr Metab Care 2:493498. https://doi.org/10.1097/00075197-199911000-00011

Dröge W, Holm E (1997) Role of cysteine and glutathione in HIV infection and other diseases associated with muscle wasting and immunological dysfunction. FASEB J 11:1077-1089 
Dröge W, Eck HP, Gmünder H, Mihm S (1991) Modulation of lymphocyte functions and immune responses by cysteine and cysteine derivatives. Am J Med. https://doi.org/10.1016/00029343(91)90297-B

Fermo I, D'Angelo SV, Paroni R et al (1995) Prevalence of moderate hyperhomocysteinemia in patients with early-onset venous and arterial occlusive disease. Ann Intern Med 123:747-753

Fukagawa NK (2006) Sparing of methionine requirements: evaluation of human data takes sulfur amino acids beyond protein. J Nutr 136:1676s-1681s

Garlick PJ (2006) Toxicity of methionine in humans. J Nutr 136:1722S-1725S

Griffith OW (1987) Mammalian sulfur amino acid metabolism: an overview. Methods Enzymol 143:366-376

Grimble RF (2005) Immunonutrition. Curr Opin Gastroenterol 21:216222. https://doi.org/10.1097/01.mog.0000153360.90653.82

Jackson AA, Gibson NR, Lu Y, Jahoor F (2004) Synthesis of erythrocyte glutathione in healthy adults consuming the safe amount of dietary protein. Am J Clin Nutr 80:101-107

Jones DP (2004) Cysteine/cystine couple is a newly recognized node in the circuitry for biologic redox signaling and control. FASEB J. https://doi.org/10.1096/fj.03-0971fje

Jones CY, Tang AM, Forrester JE et al (2006) Micronutrient levels and HIV disease status in HIV-infected patients on highly active antiretroviral therapy in the Nutrition for Healthy Living cohort. J Acquir Immune Defic Syndr 43:475-482. https://doi. org/10.1097/01.qai.0000243096.27029.fe

Kozich V, Kraus E, De Franchis R et al (1995) Hyperhomocysteinemia in premature arterial disease: examination of cystathionine $\beta$-synthase alleles at the molecular level. Hum Mol Genet 4:623629. https://doi.org/10.1093/hmg/4.4.623

Lanzillotti JS, Tang AM (2005) Micronutrients and HIV disease: a review pre- and post-HAART. Nutr Clin Care 8:16-23

Lyons J, Rauh-Pfeiffer A, Yu YM et al (2000) Blood glutathione synthesis rates in healthy adults receiving a sulfur amino acidfree diet. Proc Natl Acad Sci USA 97:5071-5076. https://doi. org/10.1073/pnas.090083297

Meister A (1988) Glutathione metabolism and its selective modification. J Biol Chem 263:17205-17208

Nguyen D, Hsu JW, Jahoor F, Sekhar RV (2014) Effect of increasing glutathione with cysteine and glycine supplementation on mitochondrial fuel oxidation, insulin sensitivity, and body composition in older HIV-infected patients. J Clin Endocrinol Metab 99:169-177. https://doi.org/10.1210/jc.2013-2376

Paroni R, De Vecchi E, Cighetti G et al (1995) HPLC with o-phthalaldehyde precolumn derivatization to measure total, oxidized, and protein-bound glutathione in blood, plasma, and tissue. Clin Chem 41:448-454

Patrick L (2000) Nutrients and HIV: part three- $N$-acetylcysteine, alpha-lipoic acid, L-glutamine, and L-carnitine. Altern Med Rev 5:290-305
Qureshi GA, Qureshi AR (1989) Determination of free amino acids in biological samples: Problems of quantitation. J Chromatogr Biomedical Appl 491:281-289

Radtke KK, Coles LD, Mishra U et al (2012) Interaction of $N$-acetylcysteine and cysteine in human plasma. J Pharm Sci 101:46534659. https://doi.org/10.1002/jps.23325

Raftos JE, Whillier S, Chapman BE, Kuchel PW (2007) Kinetics of uptake and deacetylation of $N$-acetylcysteine by human erythrocytes. Int J Biochem Cell Biol 39:1698-1706. https://doi. org/10.1016/j.biocel.2007.04.014

Rose WC (1938) The nutritive significance of the amino acids. Physiol Rev 18:109-136

Sbrana E, Paladini A, Bramanti E et al (2004) Quantitation of reduced glutathione and cysteine in human immunodeficiency virusinfected patients. Electrophoresis 25:1522-1529

Sekhar RV, Patel SG, Guthikonda AP et al (2011) Deficient synthesis of glutathione underlies oxidative stress in aging and can be corrected by dietary cysteine and glycine supplementation. Am J Clin Nutr 94:847-853. https://doi.org/10.3945/ajcn.110.003483

Stipanuk MH (2004) Sulfur amino acid metabolism: pathways for production and removal of homocysteine and cysteine. Annu Rev Nutr 24:539-577. https://doi.org/10.1146/annurev.nutr.24.01200 3.132418

Tousoulis D, Antoniades C, Marinou K et al (2008) Methionine-loading rapidly impairs endothelial function, by mechanisms independent of endothelin-1: evidence for an association of fasting total homocysteine with plasma endothelin-1 levels. J Am Coll Nutr 27:379-386

Triguero A, Barber T, García C et al (1997) Liver intracellular $\mathrm{L}$-cysteine concentration is maintained after inhibition of the trans-sulfuration pathway by propargylglycine in rats. Br J Nutr 78:823-831 (S0007114597001852 [pii])

Ubbink JB, Hayward Vermaak WJ, Bissbort S (1991) Rapid high-performance liquid chromatographic assay for total homocysteine levels in human serum. J Chromatogr 565:441-446

van der Griend R, Biesma DH, Banga JD (2002) Postmethionine-load homocysteine determination for the diagnosis hyperhomocysteinaemia and efficacy of homocysteine lowering treatment regimens. Vasc Med 7:29-33. https://doi.org/10.1191/1358863x02 vm407ra

Viñ JR, Giménez A, Corbacho A et al (2001) Blood sulfur-amino acid concentration reflects an impairment of liver transsulfuration pathway in patients with acute abdominal inflammatory processes. $\mathrm{Br}$ J Nutr 85:173-178. https://doi.org/10.1079/BJN2000237

Zhou J, Coles LD, Kartha RV et al (2015) Intravenous administration of stable-labeled $\mathrm{N}$-acetylcysteine demonstrates an indirect mechanism for boosting glutathione and improving redox status. J Pharm Sci 104:2619-2626. https://doi.org/10.1002/jps.24482 